\title{
Black Phosphorus Nanosheets as a Robust Delivery Platform for Cancer Theranostics
}

\author{
Dr. Wei Tao, \\ Graduate School at Shenzhen, Tsinghua University, Shenzhen 518055, and School of Life \\ Sciences, Tsinghua University, Beijing 100084, China. Shenzhen Key Laboratory of two- \\ dimensional materials and devices (SKTMD), SZU-NUS Collaborative Innovation Center for \\ Optoelectronic Science and Technology, and Key Laboratory of Optoelectronic Devices and \\ Systems of Ministry of Education and Guangdong Province, Shenzhen University, Shenzhen \\ 518060, P.R. China. Brigham and Women's Hospital, Harvard Medical School, 75 Francis Street, \\ Boston, MA 02115, USA
}

\section{Xianbing Zhu,}

Graduate School at Shenzhen, Tsinghua University, Shenzhen 518055, and School of Life Sciences, Tsinghua University, Beijing 100084, China

\section{Xinghua Yu,}

Graduate School at Shenzhen, Tsinghua University, Shenzhen 518055, China

\section{Dr. Xiaowei Zeng,}

Graduate School at Shenzhen, Tsinghua University, Shenzhen 518055, and School of Life Sciences, Tsinghua University, Beijing 100084, China

\section{Dr. Quanlan Xiao,}

Shenzhen Key Laboratory of two-dimensional materials and devices (SKTMD), SZU-NUS Collaborative Innovation Center for Optoelectronic Science and Technology, and Key Laboratory of Optoelectronic Devices and Systems of Ministry of Education and Guangdong Province, Shenzhen University, Shenzhen 518060, P.R. China

\section{Dr. Xudong Zhang,}

Graduate School at Shenzhen, Tsinghua University, Shenzhen 518055, and School of Life Sciences, Tsinghua University, Beijing 100084, China

\section{Xiaoyuan Ji,}

Brigham and Women's Hospital, Harvard Medical School, 75 Francis Street, Boston, MA 02115, USA

\section{Dr. Xusheng Wang,}

Graduate School at Shenzhen, Tsinghua University, Shenzhen 518055, and School of Life Sciences, Tsinghua University, Beijing 100084, China

\section{Prof. Jinjun Shi,}

Brigham and Women's Hospital, Harvard Medical School, 75 Francis Street, Boston, MA 02115, USA 
Prof. Han Zhang, and

Shenzhen Key Laboratory of two-dimensional materials and devices (SKTMD), SZU-NUS

Collaborative Innovation Center for Optoelectronic Science and Technology, and Key Laboratory

of Optoelectronic Devices and Systems of Ministry of Education and Guangdong Province, Shenzhen University, Shenzhen 518060, P.R. China

Prof. Lin Mei

Graduate School at Shenzhen, Tsinghua University, Shenzhen 518055, and School of Life Sciences, Tsinghua University, Beijing 100084, China

\section{Abstract}

2D black phosphorus (BP) nanomaterials as a delivery platform is presented. The endocytosis pathways and biological activities of PEGylated BP nanosheets in cancer cells are revealed for the first time. Finally, a triple-response combined therapy strategy is achieved by PEGylated BP nanosheets, showing a promising and enhanced antitumor effect.

\section{Graphical Abstract}

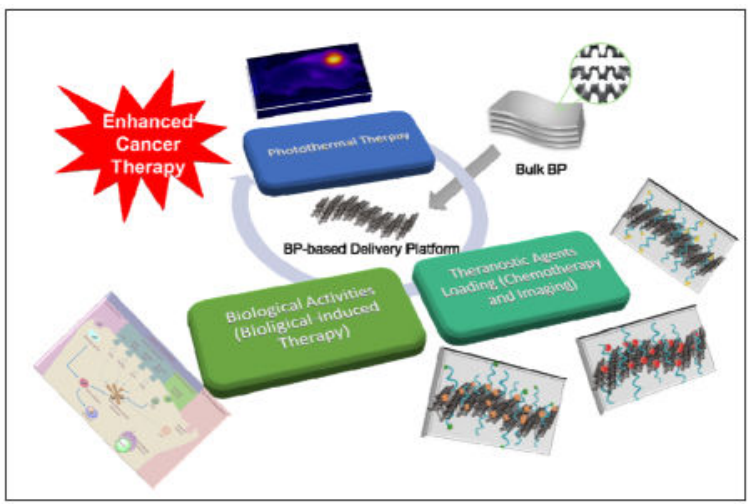

\section{Keywords}

Black Phosphorus; Nanosheet; Theranostic Delivery Platform; Enhanced Cancer Therapy

The wave of research on two-dimensional (2D) nanomaterials began in 2004, when the first graphene was exfoliated from graphite by a mechanical cleavage technique. ${ }^{[1,2]}$ Over the past decade, an increasing number of monolayer/multilayer 2D nanomaterials with various unique physical and chemical properties have been widely studied and have shown promising applications in optoelectronics, electronics, energy storage and conversion, and biomedicine (e.g., therapeutic delivery, imaging/diagnosis, and biosensors). ${ }^{[3,4]}$ Examples include transition-metal dichalcogenides (TMDs), ${ }^{[5]}$ covalent-organic frameworks $(\mathrm{COFs}),{ }^{[6]}$ hexagonal boron nitride (h-BN), ${ }^{[7]}$ metal-organic frameworks (MOFs), ${ }^{[8]}$ layered double hydroxides (LDHs), ${ }^{[9]}$ and black phosphorus (BP). ${ }^{[10]}$ Among the large family of 2D nanomaterials, BP has recently attracted enormous attention due to its distinct structure with corrugated planes of $\mathrm{P}$ atoms, which are connected by strong intralayer P-P bonding and weak interlayer Van Der Waals forces. ${ }^{[11]}$ By breaking down the weak interlayer 
interactions, the bulk BP can be exfoliated into thin BP sheets with a few layers or even a monolayer. A layer-dependent bandgap of $0.3 \mathrm{eV}$ to approximately $2 \mathrm{eV}$, as well as highly accurate optical-response properties and an anisotropic charge transport, can be achieved by controlling the structure, leading to fascinating electronic and photoelectronic applications of BP. ${ }^{[12]}$

More recently, a few studies have also shown the potential of BP nanomaterials in biomedical applications. For instance, ultrathin BP nanosheets (NSs) could generate efficient single oxygen and serve as effective photodynamic therapy (PDT) agents. ${ }^{[13]}$ Because of the high extinction coefficient and photothermal conversion efficiency, the latest reports have shown that there is promising potential for BP quantum dots (QDs) and BP nanoparticles (NPs) as a photothermal therapy (PTT) of cancer. ${ }^{[14,15]}$ The cytotoxicity of BP nanomaterials was also preliminarily studied in these reports, showing no observable toxicity in various cells. ${ }^{[13-16]}$ However, despite the few pioneering studies, the potential of using BP-based nanomaterials as a theranostic delivery platform has not been demonstrated. We expect that the BP NSs may have great possibility to enable efficient loading of theranostic agents, similar to graphene, $\mathrm{MoS}_{2}$ or other theranostic tools, because of the atomically-thin 2D structure and relatively large surface area. ${ }^{[4,17]}$ In addition, the biological activities of BP-based nanomaterials are closely related with the fate (e.g., pathway, location, biocompatibility, and biodegradability) of these nano-systems in cancer cells, playing a crucial role in an essential understanding of BP and other emerging 2D nanomaterials in cancer cells. Nevertheless, research on the biological activities of BP-based nanomaterials has also not been reported until now.

Herein, we designed a theranostic delivery platform based on 2D BP NSs (Figure 1a), studied their biological activities by screening the endocytosis pathways in tumor cells, and finally applied this BP delivery platform in cancer theranostics. BP NSs were synthesized by a modified mechanical exfoliation method from bulk BP and were then functionalized with positively charged polyethylene glycol-amine $\left(\mathrm{PEG}-\mathrm{NH}_{2}\right)$ via electrostatic adsorption to improve their biocompatibility and physiological stability. The developed PEGylated BP NSs could load theranostic agents with high efficiency, such as doxorubicin (DOX) for chemotherapy and cyanine7 (Cy7) for in vivo near-infrared (NIR) imaging. The endocytosis pathways of PEGylated BP NSs were revealed with a final concentration in lysosomes (Figure 1b). With excellent biocompatibility, DOX-loaded PEGylated BP NSs exhibited enhanced antitumor effects (i.e., photothermal-, chemo- and biological response-induced therapy) both in vitro and in vivo. Therefore, our study demonstrated the promising use of $\mathrm{BP}$ as an innovative 2D platform for theranostic delivery and revealed the biological activities of PEGylated BP NSs in cancer cells for the first time, which we expect will provide insights for deep understanding of other emerging 2D nanomaterials.

In the first set of experiments, a modified mechanical exfoliation method ${ }^{[13]}$ was adopted to prepare the 2D BP NSs (Figure S1). Although organic solvents such as N-methyl-2pyrrolidone (NMP) could also lead to good exfoliation efficiency, ${ }^{[11,18]}$ we chose oxygen molecule-free water as the solvent for the mechanical exfoliation of bulk BP into NSs via probe sonication. This solvent will avoid the potential toxicity associated with organic solvents and ensure a clean surface of the obtained NSs for medical use. Due to the electron 
screening effect, BP NSs would aggregate and precipitate, especially in the presence of salts such as phosphate-buffered saline (PBS) and cell culture medium (Figure S2a, 2b, 2e).

Therefore, after centrifugation to remove un-exfoliated BP, pure BP NSs were modified by PEG-NH $\mathrm{N}_{2}$ to enhance their stability in physiological medium. As presented in this study, the PEGylated BP NSs were observed with negligible agglomeration after 1 week of incubation, showing remarkable stability in PBS and cell culture medium (Figure S2a, 2c-d, 2f-g). The chemical composition and crystal structure of BP-PEG NSs were further confirmed by X-ray diffractometry (XRD) and X-ray photoelectron spectroscopy (XPS). As shown in Figure S3, the BP-PEG NSs could be indexed into orthorhombic BP consistent with JCPDS No.

73-1358. ${ }^{[13,15]}$ No other elements were detected except $\mathrm{P}, \mathrm{C}$ and $\mathrm{O}$, indicating the high purity of the final product (Figure S4a). The two strong peaks at $\sim 129.9 \mathrm{eV}$ and $\sim 130.7 \mathrm{eV}$ are the $2 \mathrm{p} 3 / 2$ and $2 \mathrm{p} 1 / 2$ orbitals of zero-valent $\mathrm{P}$ in the $\mathrm{P} 2 \mathrm{p}$ spectrum, respectively (Figure $\mathrm{S} 4 \mathrm{~b})$. The weak peak at $\sim 134.0 \mathrm{eV}$ is the signal of oxidized phosphorus $\left(\mathrm{P}^{3+}\right.$ and $\left.\mathrm{P}^{5+}\right)$ due to the minor degradation on the surface of NSs as previously reported. ${ }^{[13]}$ The hydrodynamic size of BP-PEG NSs in PBS and fetal bovine serum (FBS) was further monitored over a span of one week (Figure S5). This study further confirmed the stability of BP-PEG NSs, as their sizes had no significant change. The zeta potential of BP-based NSs were determined (Figure S6), and a relatively reduced surface charge of BP-PEG NSs was observed compared with that in a previous study $(-10.3 \mathrm{mV}$ in our study $v s .-35.4 \mathrm{mV}),{ }^{[15]}$ possibly attributable to the inhibition of the partial oxidation of BP (formation of phosphate groups) via this modified method.

Transmission electron microscopy (TEM) and atomic force microscopy (AFM) were used to characterize the surface morphology of BP NSs pre- and post-PEGylation (Figure 2a-f). The average size of BP NSs was $\sim 120 \mathrm{~nm}$ (TEM and AFM), and the average height of BP NSs was $\sim 1-2 \mathrm{~nm}$ (AFM). For BP-PEG NSs, the average size was $\sim 100 \mathrm{~nm}$ (TEM and AFM) and the average height was $\sim 2-3 \mathrm{~nm}$. The minimal decrease in the size of BP-PEG NSs was caused by additional sonication during the coating of PEG- $\mathrm{NH}_{2}$, and the slight increase in thickness could be attributed to a few amount of PEG that was coated on the NS surface. ${ }^{[19,20]}$ To further demonstrate that PEG-NH $\mathrm{NH}_{2}$ or other functionalization-modified PEG-NH $\mathrm{N}_{2}$ such as folic acid (FA)-PEG-NH $\mathrm{N}_{2}$ could be successfully coated on the surface of BP NSs, we tested the Fourier transform infrared spectra (FT-IR) using scanning transmission electron microscopy (STEM) with energy dispersive X-ray spectroscopy (EDS) mapping of elements. With an absorption band at $\sim 2900 \mathrm{~cm}^{-1}$ that was attributable to the $\mathrm{CH}$ vibration in the PEG segment and characteristic stretching vibration at $~ 1637$ to 1653 $\mathrm{cm}^{-1}$ from the amide bonding within FA structure, the coating of PEG-NH $\mathrm{N}_{2}$ or FA-PEG$\mathrm{NH}_{2}$ was confirmed (Figure $\mathrm{S} 7$ ). The feasibility of this coating method was also validated by STEM-EDS showing the excellent co-localization of four different elements $(\mathrm{C}, \mathrm{O}$ and $\mathrm{N}$ elements from the surface coating FA-PEG-NH $\mathrm{N}_{2}$, and P element from BP) in Figure 2g. Raman spectral analysis, which is an effective method for sample identification through detailed rotational and vibrational modes, was performed to verify the structure of PEGylated BP NSs (Figure S8). The Raman spectra of exfoliated BP NSs showed nearly the same peaks located at $\sim 363.6,439.3$, and $467.4 \mathrm{~cm}^{-1}$ with those of bulk BP reported previously, ${ }^{[13]}$ which correspond to $\mathrm{A}_{\mathrm{g}}{ }^{1}, \mathrm{~B}_{2 \mathrm{~g}}$, and $\mathrm{A}_{\mathrm{g}}{ }^{2}$ modes of $\mathrm{BP}$, respectively, suggesting that all prepared NSs did not have structural transformations compared with the 
corresponding bulk counterpart. A slight shift toward a low wavenumber could be found in PEGylated BP NSs, which could be caused by the slight change in the ultrathin height after PEG (or functionalized PEG) coating. ${ }^{[21]}$ Similar to the targeting modified PEG- $\mathrm{NH}_{2}$ (i.e., FA-PEG-NH $\mathrm{N}_{2}$ ), the fluorescence-modified PEG- $\mathrm{NH}_{2}$ (i.e., FITC-PEG-NH $\mathrm{N}_{2}$ ) could also be coated on the surface of BP NSs with the same principle. The amount of PEG that was coated on the BP surface was $\sim 25.8 \%$ (w/w \%) of the PEGylated BP NSs that was determined by the absorbance of FITC-labeled PEG. At the end of this part, the excellent photothermal properties of BP-PEG NSs were tested and were well demonstrated (see Supporting Information).

In the next set of experiments, we tested the use of BP-PEG NSs as a novel theranostic delivery platform. Since 2D nanomaterials such as TMDs, graphene and their derivatives, which possess a relatively high surface area, have been widely reported as drug carriers to interact with various types of drug molecules, ${ }^{[2,20]}$ the potential loading abilities of BPbased materials were well worth the expectation. A BP-PEG NS solution was mixed with DOX at different feeding ratios (DOX/NSs feeding ratios: 0.6, 1.2, 1.8, 2.4, 3, 3.6, and 4.2) and then stirred overnight. The UV-vis-NIR spectra were measured to calculate the drugloading ratio of these BP-PEG NSs after removing excess-free DOX molecules (Figure 2h). As the concentrations of the DOX/NSs feeding ratios increased, the loading capacities of DOX increased almost linearly and reached a saturation level at a DOX/NSs feeding ratio of 3 (Figure 2i). Our tested conditions showed that the saturation of DOX loading was tested to be $\sim 108 \%$ and that this was noticeably higher than many conventional NP-based nanodelivery systems with a usual range of $\sim 10-30 \%$ for drug loading. ${ }^{[22]}$ Moreover, the drugrelease behavior of this novel delivery platform was further investigated in our studies. The DOX-loaded NSs (BP-PEG/DOX NSs) were dialyzed in PBS solution at different $\mathrm{pH}$ values (7.4 and 5.0). The drug-release kinetics were determined by collecting the released DOX molecules at different time intervals (Figure S12 and S13). Approximately 33.4\% of DOX was released from BP-PEG NSs at $\mathrm{pH} 5.0$ over a span of $24 \mathrm{~h}$, while $\sim 15.2 \%$ of DOX was released at $\mathrm{pH} 7.4$ in our studies. The cause of this release may be the protonation of the amino group present on the sugar moiety of DOX. An NIR laser-induced local hyperthermia stimulus for the on/off control of the DOX release from BP-PEG/DOX NSs could also be observed in this figure, leading to a higher drug release of $54.4 \%$, and may contribute to enhanced antitumor therapy. Cy7- $\mathrm{NH}_{2}$ could be loaded on the surface of BP-PEG NSs using the similar method, and the Cy7-loaded NSs used in this study were $~ 30.4 \%$ (w/w \%) of Cy7- $\mathrm{NH}_{2}$ on the NSs surface (Figure S14). Moreover, the fluorescence of loaded molecules could be quenched partially by PEGylated BP NSs, indicating strong interactions between drug molecules and BP-PEG NSs (Figure S15). ${ }^{[20]}$

After the confirmation of the in vitro safety of these PEGylated BP NSs (see Supporting Information), we next continued to evaluate the in vitro therapeutic effects of PEGylated BP NSs as single photothermal agents and as drug carriers based on their satisfactory performance. HeLa cells were selected as model cancer cells in our following studies. Through MTT assays, we could observe the excellent PTT efficiency of BP NSs and BPPEG NSs in promoting the death of cancer cells since $~ 90 \%$ of the cells were killed by only using $50 \mu \mathrm{g} / \mathrm{ml}$ BP NSs or BP-PEG NSs (while $\sim 40 \%$ for $25 \mu \mathrm{g} / \mathrm{ml}$ ) irradiated with an 808 $\mathrm{nm}$ laser at $1.0 \mathrm{~W} / \mathrm{cm}^{2}$ for $10 \mathrm{~min}$ compared with the cells without any treatment (Figure 
S18a). The results also demonstrated that PEGylation did not influence the great PTT effect of BP NSs. We subsequently tested the potential of these NSs as a drug-delivery platform for cancer chemotherapy with DOX as a model drug. BP-PEG/DOX NSs exhibited slightly reduced cytotoxicity compared with free DOX (Figure S18b), similar to many nano-delivery platforms. These results may be attributed to the relatively slower endocytosis of the nanodelivery platform and additional process of intracellular DOX release compared with free DOX molecules. In order to promote the therapeutic efficiency and construct specific targeting delivery systems, we also introduced targeting modified BP-PEG-FA NSs to act as model nano-carriers for DOX due to the specific binding ability between FA and folatereceptor over-expressed on many cancer cells, a finding that was also demonstrated by many groups as well as by our previous studies. ${ }^{[23]}$ Based on the former studies, quantitative flow cytometry (FCM) was used to further demonstrate the strong FA-mediated cancer targeting effect of BP-PEG-FA NSs. As shown in Figure S19, the cellular DOX fluorescence intensities in HeLa cells after $1 \mathrm{~h}$ of incubation with BP-PEG-FA/DOX NSs was significantly higher than BP-PEG/DOX NSs, proving a higher cellular uptake efficiency of BP-PEG-FA/DOX NSs. Furthermore, the in vitro cellular toxicity of BP-PEG-FA NSs as photothermal agents and BP-PEG-FA/DOX NSs as chemotherapy agents were both effectively enhanced compared with that of BP-PEG NSs and BP-PEG/DOX NSs, also indicating the large in vitro targeting effect of BP-PEG-FA NSs. Taken together, the results indicated that PEGylated BP NSs could be utilized to develop a versatile and functionalized delivery platform and that they are very promising for application in cancer theranostics due to the excellent photothermal efficiency, the high-loading efficiency and especially the low toxicity.

In the third set of experiments, we performed a biological study of this delivery platform in cancer cells. When the PEGylated BP NSs reached the external milieu of cancer cells through long-term circulation, they could interact with the surface of the cell plasma membrane. The interaction results in the internalization of PEGylated BP NSs through a process termed endocytosis, either clathrin-dependent or clathrin-independent. ${ }^{[24]}$ The clathrin-independent pathways are divided into 1) macropinocytosis, 2) caveolaeindependent endocytosis and 3) caveolae-dependent endocytosis. Macropinocytosis is controlled by Rab34, which is considered a biomarker of this process. The caveolaeindependent endocytosis mainly includes flotillin-, Arf6-, Cdc42- and RhoA-dependent endocytosis. ${ }^{[25]}$ Fluorescent imaging agents FITC-labeled BP-PEG (BP-PEG-FITC) NSs were employed in the screening of all the possible endocytosis pathways of these NSs. The BP-PEG-FITC NSs could be internalized by HeLa cells efficiently after $4 \mathrm{~h}$ of incubation (Figure S20). Moreover, macropinocytosis and caveolae-dependent endocytosis are involved in the entry of the BP-PEG-FITC NSs into the cells. We observed both co-localization of FITC-positive vesicles with caveolae-positive vesicles (Figure 3a) and FITC-positive vesicles with Rab34-positive vesicles (Figure 3b). However, no merge of FITC-positive vesicles with clathrin-, flotillin-, Arf6-, Cdc42- or RhoA-positive vesicles was found (Figure S21-25). These results suggested that the pathways for PEGylated BP NSs occur mainly through caveolae-dependent endocytosis and macropinocytosis but not via the clathrindependent pathway. 
In the classic endocytosis pathways, nano-systems would be transported to early endosomes and late endosomes soon afterwards and would finally be delivered to lysosomes after being internalized into the cells. Rab5 and EEA1 are widely-used markers in early endosomes, whereas Rab7 is used as a marker of late endosomes. ${ }^{[26]}$ We then verified whether our PEGylated BP NSs would be transported via this pathway by detecting the co-localization between caveolae and DsRed-Rab5 (Figure S26a) since caveolae-positive vesicles may deliver BP-PEG-FITC NSs to early endosomes and then transport them to late endosomes. As presented in our studies, BP-PEG-FITC NSs co-localized perfectly with both DsRedRab5-labeled early endosomes and DsRed-Rab7-marked late endosomes (Figure 3c and 3d). Furthermore, BP-PEG-FITC NSs merged well with the lysosomes while the Lyso-Tracker was used to mark lysosomes (Figure 3e). These results indicated that BP-PEG-FITC NSs were taken up by cells through caveolae-dependent endocytosis and were then transported to early endosomes and late endosomes, and finally degraded in lysosomes through the classic endocytosis pathway. However, in the macropinocytosis pathway, we did not find that DsRed-Rab34 co-localized with EEA1-labeled early endosomes (Figure S26b). In contrast, DsRed-Rab34 co-localized well with DsRed-Rab7-labeled late endosomes (Figure S26c). These data implied that macropinocytosis (Rab34-labeled) $\rightarrow$ late endosomes (Rab7labeled) $\rightarrow$ lysosomes could be a novel endocytosis pathway in the turnover of BP-PEGFITC NSs (Figure 1b).

After revealing the endocytosis pathways, we then studied the biological activities of PEGylated BP NSs and focused on autophagy studies since it could sequester most nanomaterials and transport them to lysosomes for degradation. ${ }^{[27]}$ To test the relationship between PEGylated BP NSs and autophagy, LC3-II protein and P62 protein were used as autophagy marker proteins. ${ }^{[28]}$ After incubation with BP-PEG-FITC NSs for $24 \mathrm{~h}$, the LC3II protein levels were increased and P62 protein levels were reduced in the cells (Figure 3f). Furthermore, autophagosomes were significantly increased within the cells as shown in Figure 3g, indicating that PEGylated BP NSs could induce autophagy in cancer cells. We further found that P62, which is a marker of sequestosome1 (SQSTM1) and an adapter molecule that selectively recognized and bound the substrates of autophagy, co-localized with BP-PEG-FITC NSs (Figure S27a). LC3, which is a marker of autophagosomes, could interact directly with P62/SQSTM1 and capture P62 on the isolation membrane. We observed co-localization between P62-positive sequestosome1 and LC3-positive autophagosomes (Figure S27b). As expected, we also perceived that BP-PEG-FITC NSs containing vesicles merged perfectly with LC3-positive autophagosomes (Figure S28a). P62-positive sequestosome1 selects the target, and LC3-positive autophagosomes select P62. Finally, autophagosomes containing BP-PEG-FITC NSs translocated to fuse with lysosomes for degradation (Figure S28b). These results advocated P62-positive sequestosome1 targeted BP-PEG-FITC NSs and transported them to LC3-positive autophagosomes. The autophagosomes then delivered them to lysosomes. Therefore, in addition to classic endocytosis pathways, autophagy was demonstrated to be involved in the degradation of PEGylated BP NSs.

Since the PEGylated BP delivery platform was finally concentrated in the lysosomes for degradation, we may further enhance the therapeutic effect and reduce the dose of PEGylated BP NSs by inhibiting the activities of lysosomes and autophagy. To verify this, 
chloroquine (CQ), which is a lysosome tropic agent that prevents endosomal acidification, blocks the fusion of autophagosomes with lysosomes and disrupts lysosomes, ${ }^{[29]}$ which was utilized in this part of our studies. We incubated pre-treated HeLa cells ( $30 \mu \mathrm{M}$ free CQ, 24$\mathrm{h}$ incubation) with BP-PEG NSs and BP-PEG-FA NSs at low concentrations (5, 10, and 25 $\mu \mathrm{g} / \mathrm{ml}$ ), as well as HeLa cells without pre-treated (i.e., free from CQ). After $4 \mathrm{~h}$ of incubation with these NSs, HeLa cells were washed with PBS 3 times, placed into DMEM, and then irradiated with an $808-\mathrm{nm}$ NIR laser at $1.0 \mathrm{~W} / \mathrm{cm}^{2}$ for $10 \mathrm{~min}$. As expected, the cell viability decreased in the presence of CQ molecules in both the BP-PEG NSs and BP-PEG-FA NSs groups compared with the CQ-free BP-PEG NSs group and BP-PEG-FA NSs (Figure 3h). Moreover, free CQ did not show observable toxicity to HeLa cells at our tested concentration. Thus, the therapeutic effects could be caused by the inhibition of lysosomes and blockade of the fusion between autophagosomes and lysosomes under the effect of CQ, which may reduce the degradation of PEGylated BP NSs. We further introduced DOX-load NSs for enhanced therapy of cancer in vitro. Even at a low DOX concentration $(5 \mu \mathrm{g} / \mathrm{ml})$, the cell viability in groups treated with three factors (NIR, CQ, and DOX) was significantly decreased because of the synergistic effect compared with that in previous groups. Until now, we confirmed the excellent in vitro therapeutic effect of these PEGylated BP NSs, which were attributed to the PTT effect triggered by the NIR irradiation of BP-based NSs, DOX-induced chemotherapy, and CQ-mediated inhibition of lysosomes and autophagy.

In the final set of the experiments, we carried out animal assays to test the possibilities of the PEGylated BP theranostic delivery platform for in vivo application as inspired by so many exciting in vitro results. We first studied the in vivo distribution and tumor accumulation of our BP delivery platform via Cy7-loaded PEGylated BP NSs. After intravenous (i.v.) injection of BP-PEG/Cy7 NSs and BP-PEG-FA/Cy7 NSs at different time frames $(1 \mathrm{~h}, 12 \mathrm{~h}$ and $24 \mathrm{~h}$ ), a whole-animal NIR imaging approach was utilized to monitor the dynamic change of fluorescent PEGylated BP NSs and their tumor accumulation (Figure 4a and S29). As presented in the results, Cy7-loaded PEGylated BP NSs were distributed throughout the whole body with strong fluorescence signals at $1 \mathrm{~h}$ post-injection. At $12 \mathrm{~h}$ post-injection, the fluorescence signals in both groups decreased due to the clearance of the dye by the body. Meanwhile, more signals were detected in the tumor tissues of the BP-PEG-FA NSs group than in those of the BP-PEG NSs group. At $24 \mathrm{~h}$ post-injection, the fluorescence signals continued to wane in both groups. However, strong fluorescence signals could be observed in the tumor tissues of both groups, demonstrating good tumor accumulation of PEGylated BP NSs through the blood circulation and an enhanced permeability retention (EPR) effect. Moreover, stronger signals of the BP-PEG-FA/Cy7 group than those of the BP-PEG/Cy7 group could further demonstrate the in vivo targeting effect of BP-PEG-FA NSs, indicating versatile modification such as the targeting effect being very feasible with our PEGylated BP NSs. We further confirmed the good in vivo tumor accumulation of our PEGylated BP NSs and their versatile ability to be in vivo targeting via the ex vivo study of excised organs at 24 $\mathrm{h}$ post-injection (Figure $4 \mathrm{~b}$ and $4 \mathrm{c}$ ). The fluorescence signals remained majorly in the liver and kidney because Cy7 is metabolized throughout the liver (liver Kupffer cells play an important role in the uptake and degradation in extra-phagocytosis) and excreted through the kidney. The signals in the lung could be caused by the mechanical retention of larger-sized 
NSs in lung capillaries. These biodistribution results were in good agreement with the excellent long circulation confirmed by pharmacokinetic studies (Figure S30).

Based on the in vivo biological response (see Supporting Information) and enhanced in vitro therapeutic effects, we carried out an in vivo antitumor study to validate the enhanced therapy of cancer using our PEGylated BP theranostic delivery platform. BP-PEG-FA NSs were chosen as the model NSs considering their in vivo targeting effect. The tumor-bearing nude mice were treated with Group 1: saline (control), Group 2: CQ (biological responseinduced therapy control), Group 3: DOX (chemotherapy control), Group 4: BP-PEGFA/DOX NSs (chemotherapy with delivery platform group), Group 5: BP-PEG-FA NSs + NIR (PTT group), Group 6: BP-PEG-FA NSs with i.t.-injected CQ + NIR (PTT + biological response-induced therapy group), and Group 7: BP-PEG-FA/DOX NSs + NIR with i.t.injected CQ (combined triple-response therapy group: chemotherapy + PTT + biological response induced therapy) at the same dose. Groups 5, 6 and 7 were treated with an 808-nm laser at $1 \mathrm{~W} / \mathrm{cm}^{2}$ for $10 \mathrm{~min}$ at tumor sites after $24-\mathrm{h}$ injection. The tumor volumes were calculated based on the width and length of the tumors that were measured every 2 days during the 2-week treatment (Figure 4d). As displayed in our studies, compared with saline, Groups 3-7 all showed decreasing tumor growth in mice. A better therapeutic effect could be observed in Groups 4 and 5 compared with Group 3 (clinical chemotherapy drug), indicating the promising application of PEGylated BP NSs as drug carriers and PTT agents. Moreover, a significantly therapeutic effect could be found in both Groups 6 and 7, demonstrating that the enhanced antitumor effect of PEGylated BP NSs could be achieved by this combined therapy strategy. Group 7 showed the best therapeutic effect, which was in good agreement with the in vitro toxicity results. CQ seemed to minimally inhibit tumors compared with saline, indicating that the enhanced therapy effect could be caused by inhibiting the degradation of the BP-based NSs. Meanwhile, we found that the body weights of nude mice were not significantly affected, demonstrating that there were no acute side effects in our combined therapy (Figure S32). At the end of the two-week treatment, all nude mice were euthanized and the tumors were collected, clearly showing the excellent therapeutic effect of our PEGylated BP NSs in the enhanced therapy of cancer (Figure 4e). No tissue damage could be found in the major organs in any experimental group as assessed by H\&E staining (Figure 4f). Moreover, no observable side effect or toxicity of our PEGylated BP NSs was found even at a higher dose of $10 \mathrm{mg} / \mathrm{kg}$ in the in vivo toxicity studies (see Supporting Information), indicating the promising in vivo application of this BP theranostic delivery platform. However, many more systematic studies still need to be performed before future clinical studies of these PEGylated BP NSs, as well as some bioand immune-compatibility studies. ${ }^{[30]}$

In summary, we have demonstrated the promising application of BP NSs as a robust delivery platform for the first time, thus opening an exciting and new research point of 2D BP nanomaterials as a delivery vehicle. Furthermore, we have revealed the biological activities and screened the endocytosis pathways of PEGylated BP NSs in cancer cells for the first time, providing guidance for the essential understanding of $\mathrm{BP}$ and other emerging $2 \mathrm{D}$ nanomaterials for cancer theranostics. A triple-response combined therapy strategy was proposed with these PEGylated BP NSs for cancer treatment. Both in vitro and in vivo experiments verified the safety and enhanced antitumor effect of this platform. Finally, with 
the flexible modification with functionalized PEG- $\mathrm{NH}_{2}$ and great biosafety, these BP NSs could provide a robust platform for the treatment of cancer.

\section{Supplementary Material}

Refer to Web version on PubMed Central for supplementary material.

\section{Acknowledgments}

We acknowledge the financial support from the National Institutes of Health (NIH) Grants (R00CA160350 and CA200900), Tsinghua Scholarship for Overseas Graduate Studies (2013159), National Natural Science Foundation of China (61435010 and 31270019), Guangdong Natural Science Funds for Distinguished Young Scholar (2014A030306036), Natural Science Foundation of Guangdong Province (2016A030310023, 2015A030313848), Science and Technology Planning Project of Guangdong Province (2016A020217001), Science and Technology Innovation Commission of Shenzhen (KQTD2015032416270385, JCYJ20150625103619275, JCYJ20150430163009479, JCYJ20160301152300347), Natural Science Foundation of SZU (827/000010). We are also grateful for Dr. Ling Ji's assistance with the blood routine test (BRT) at Peking University Shenzhen Hospital. The Administrative Committee on Animal Research in Tsinghua University approved the protocols for all animal assays in this paper. W.T., X.Z. and X.Y. contributed equally to this work.

\section{References}

1. Novoselov KS, Geim AK, Morozov SV, Jiang D, Zhang Y, Dubonos SV, Grigorieva IV, Firsov AA. Science. 2004; 306:666. [PubMed: 15499015] Zhang H. ACS Nano. 2015; 9:9451. [PubMed: 26407037] Zhang Y, Tang TT, Girit C, Hao Z, Martin MC, Zettl A, Crommie MF, Shen YR, Wang F. Nature. 2009; 459:820. [PubMed: 19516337] Peng X, Peng L, Wu C, Xie Y. Chem Soc Rev. 2014; 43:3303. [PubMed: 24614864] Li X, Zhu J, Wei B. Chem Soc Rev. 2016Cao X, Tan C, Zhang X, Zhao W, Zhang H. Adv Mater. 2016

2. Chen Y, Tan C, Zhang H, Wang L. Chem Soc Rev. 2015; 44:2681. [PubMed: 25519856]

3. Huang X, Tan C, Yin Z, Zhang H. Adv Mater. 2014; 26:2185. [PubMed: 24615947] Kurapati R, Kostarelos K, Prato M, Bianco A. Advanced Materials. 2016 n/a. Yang G, Zhu C, Du D, Zhu J, Lin Y. Nanoscale. 2015; 7:14217. [PubMed: 26234249]

4. Chimene D, Alge DL, Gaharwar AK. Adv Mater. 2015; 27:7261. [PubMed: 26459239]

5. Qian X, Liu J, Fu L, Li J. Science. 2014; 346:1344. [PubMed: 25504715]

6. Lin S, Diercks CS, Zhang YB, Kornienko N, Nichols EM, Zhao Y, Paris AR, Kim D, Yang P, Yaghi OM, Chang CJ. Science. 2015; 349:1208. [PubMed: 26292706]

7. Tran TT, Bray K, Ford MJ, Toth M, Aharonovich I. Nat Nanotechnol. 2016; 11:37. [PubMed: 26501751] Liu Z, Ma L, Shi G, Zhou W, Gong Y, Lei S, Yang X, Zhang J, Yu J, Hackenberg KP, Babakhani A, Idrobo JC, Vajtai R, Lou J, Ajayan PM. Nat Nanotechnol. 2013; 8:119. [PubMed: 23353677]

8. Han SS, Mendoza-Cortes JL, Goddard WA 3rd. Chem Soc Rev. 2009; 38:1460. [PubMed: 19384448]

9. Sideris PJ, Nielsen UG, Gan Z, Grey CP. Science. 2008; 321:113. [PubMed: 18599785]

10. Xia F, Wang H, Jia Y. Nat Commun. 2014; 5:4458. [PubMed: 25041752] Ryder CR, Wood JD, Wells SA, Yang Y, Jariwala D, Marks TJ, Schatz GC, Hersam MC. Nat Chem. 2016 advance online publication.

11. Yasaei P, Kumar B, Foroozan T, Wang C, Asadi M, Tuschel D, Indacochea JE, Klie RF, SalehiKhojin A. Adv Mater. 2015; 27:1887. [PubMed: 25645510]

12. Ling X, Wang H, Huang S, Xia F, Dresselhaus MS. Proc Natl Acad Sci U S A. 2015; 112:4523. [PubMed: 25820173] Li L, Yu Y, Ye GJ, Ge Q, Ou X, Wu H, Feng D, Chen XH, Zhang Y. Nat Nanotechnol. 2014; 9:372. [PubMed: 24584274] Das S, Zhang W, Demarteau M, Hoffmann A, Dubey M, Roelofs A. Nano Lett. 2014; 14:5733. [PubMed: 25111042]

13. Wang H, Yang X, Shao W, Chen S, Xie J, Zhang X, Wang J, Xie Y. J Am Chem Soc. 2015; 137:11376. [PubMed: 26284535] 
14. Sun Z, Xie H, Tang S, Yu XF, Guo Z, Shao J, Zhang H, Huang H, Wang H, Chu PK. Angewandte Chemie. 2015; 127:11688.

15. Sun C, Wen L, Zeng J, Wang Y, Sun Q, Deng L, Zhao C, Li Z. Biomaterials. 2016; 91:81. [PubMed: 27017578]

16. Latiff NM, Teo WZ, Sofer Z, Fisher AC, Pumera M. Chemistry - A European Journal. 2015; 21:13991.

17. Orecchioni M, Cabizza R, Bianco A, Delogu LG. Theranostics. 2015; 5:710. [PubMed: 25897336] Yang K, Feng L, Shi X, Liu Z. Chemical Society Reviews. 2013; 42:530. [PubMed: 23059655]

18. Kang J, Wood JD, Wells SA, Lee JH, Liu X, Chen KS, Hersam MC. ACS Nano. 2015; 9:3596. [PubMed: 25785299]

19. Cheng L, Liu J, Gu X, Gong H, Shi X, Liu T, Wang C, Wang X, Liu G, Xing H, Bu W, Sun B, Liu Z. Advanced Materials. 2014; 26:1886. [PubMed: 24375758]

20. Liu T, Wang C, Gu X, Gong H, Cheng L, Shi X, Feng L, Sun B, Liu Z. Advanced Materials. 2014; 26:3433. [PubMed: 24677423]

21. Favron A, Gaufres E, Fossard F, Phaneuf-L'Heureux AL, Tang NY, Levesque PL, Loiseau A, Leonelli R, Francoeur S, Martel R. Nat Mater. 2015; 14:826. [PubMed: 26006004] Zhang X, Xie H, Liu Z, Tan C, Luo Z, Li H, Lin J, Sun L, Chen W, Xu Z, Xie L, Huang W, Zhang H. Angew Chem Int Ed Engl. 2015; 54:3653. [PubMed: 25649505]

22. Tao W, Zeng X, Wu J, Zhu X, Yu X, Zhang X, Zhang J, Liu G, Mei L. Theranostics. 2016; 6:470. [PubMed: 26941841] Zeng X, Tao W, Mei L, Huang L, Tan C, Feng SS. Biomaterials. 2013; 34:6058. [PubMed: 23694904]

23. Tao W, Zhang J, Zeng X, Liu D, Liu G, Zhu X, Liu Y, Yu Q, Huang L, Mei L. Adv Healthc Mater. 2015; 4:1203. [PubMed: 25800699]

24. Conner SD, Schmid SL. Nature. 2003; 422:37. [PubMed: 12621426]

25. Mayor S, Pagano RE. Nat Rev Mol Cell Biol. 2007; 8:603. [PubMed: 17609668]

26. Sahay G, Alakhova DY, Kabanov AV. J Control Release. 2010; 145:182. [PubMed: 20226220]

27. Li JJ, Hartono D, Ong CN, Bay BH, Yung LY. Biomaterials. 2010; 31:5996. [PubMed: 20466420]

28. Pankiv S, Clausen TH, Lamark T, Brech A, Bruun JA, Outzen H, Overvatn A, Bjorkoy G, Johansen T. J Biol Chem. 2007; 282:24131. [PubMed: 17580304] Kabeya Y, Mizushima N, Ueno T, Yamamoto A, Kirisako T, Noda T, Kominami E, Ohsumi Y, Yoshimori T. The EMBO Journal. 2000; 19:5720. [PubMed: 11060023]

29. Zhang X, Dong Y, Zeng X, Liang X, Li X, Tao W, Chen H, Jiang Y, Mei L, Feng SS. Biomaterials. 2014; 35:1932. [PubMed: 24315578] Zhang X, Yang Y, Liang X, Zeng X, Liu Z, Tao W, Xiao X, Chen H, Huang L, Mei L. Theranostics. 2014; 4:1085. [PubMed: 25285162]

30. Orecchioni M, Menard-Moyon C, Delogu LG, Bianco A. Adv Drug Deliv Rev. 2016Orecchioni M, Jasim DA, Pescatori M, Manetti R, Fozza C, Sgarrella F, Bedognetti D, Bianco A, Kostarelos K, Delogu LG. Advanced Healthcare Materials. 2016; 5:276. [PubMed: 26687729] Pescatori M, Bedognetti D, Venturelli E, Ménard-Moyon C, Bernardini C, Muresu E, Piana A, Maida G, Manetti R, Sgarrella F, Bianco A, Delogu LG. Biomaterials. 2013; 34:4395. [PubMed: 23507086] Sailor MJ, Park JH. Advanced Materials. 2012; 24:3779. [PubMed: 22610698] 


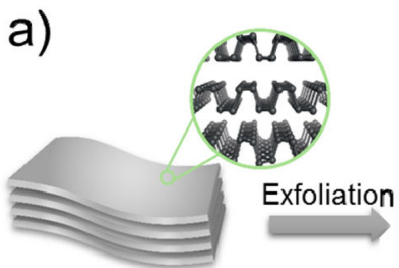

Bulk BP

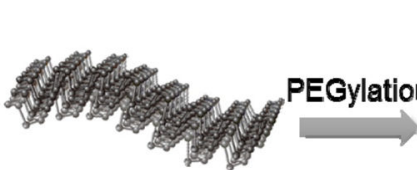

BP NSs

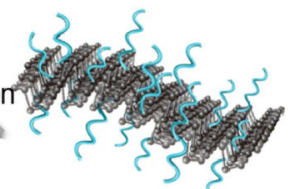

BP-PEG NSs
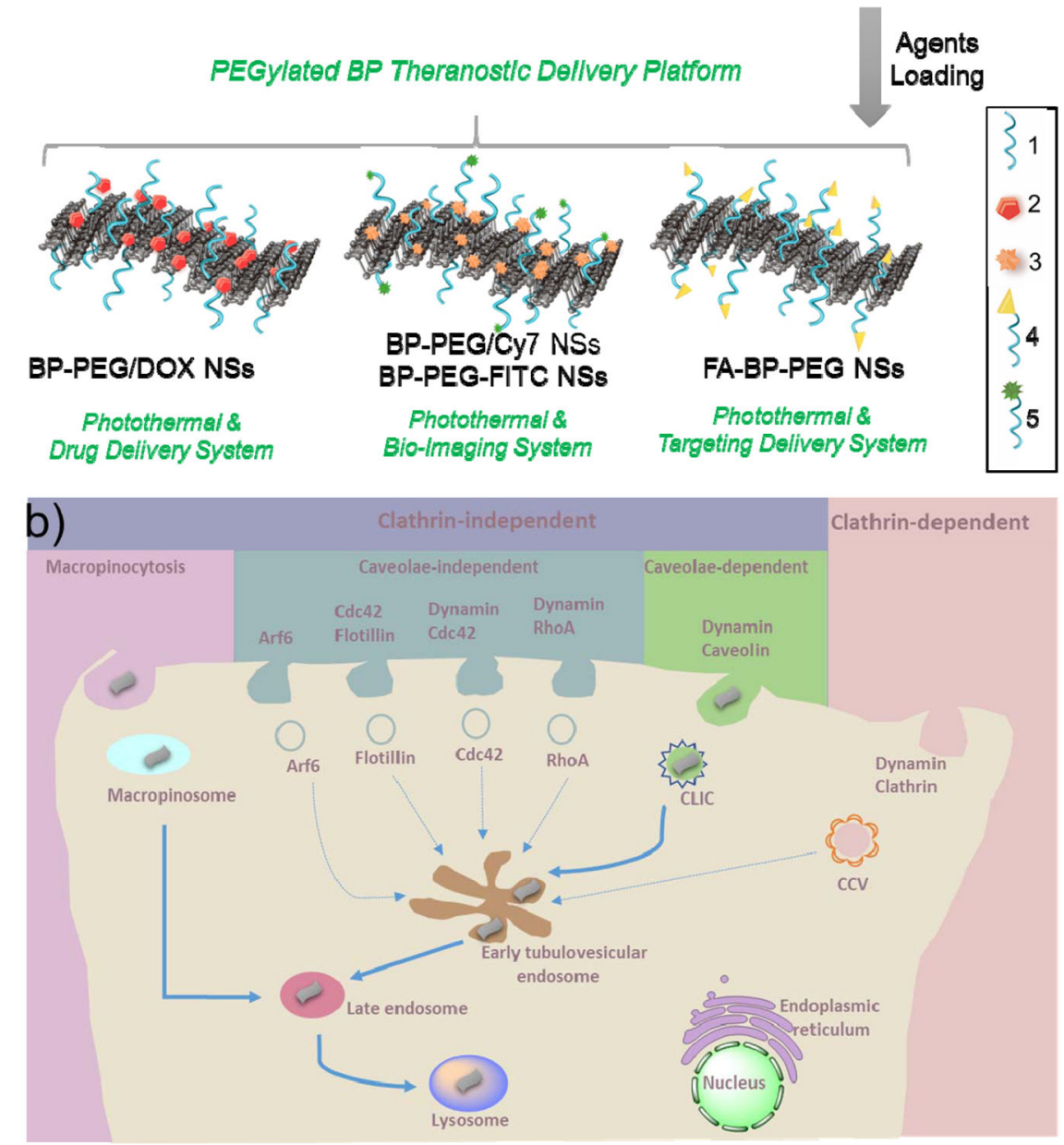

Figure 1.

(a) Schematic representation of the PEGylated BP theranostic delivery platform. 1: PEG$\mathrm{NH}_{2}$ (surface modification), 2: DOX (therapeutic agents), 3: Cy7- $\mathrm{NH}_{2}$ (NIR imaging

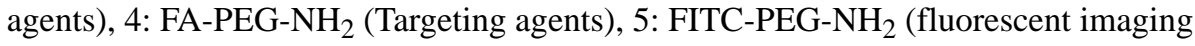
agents). (b) Screening and summary of the endocytosis pathways and biological activities of PEGylated BP NSs in cancer cells. 

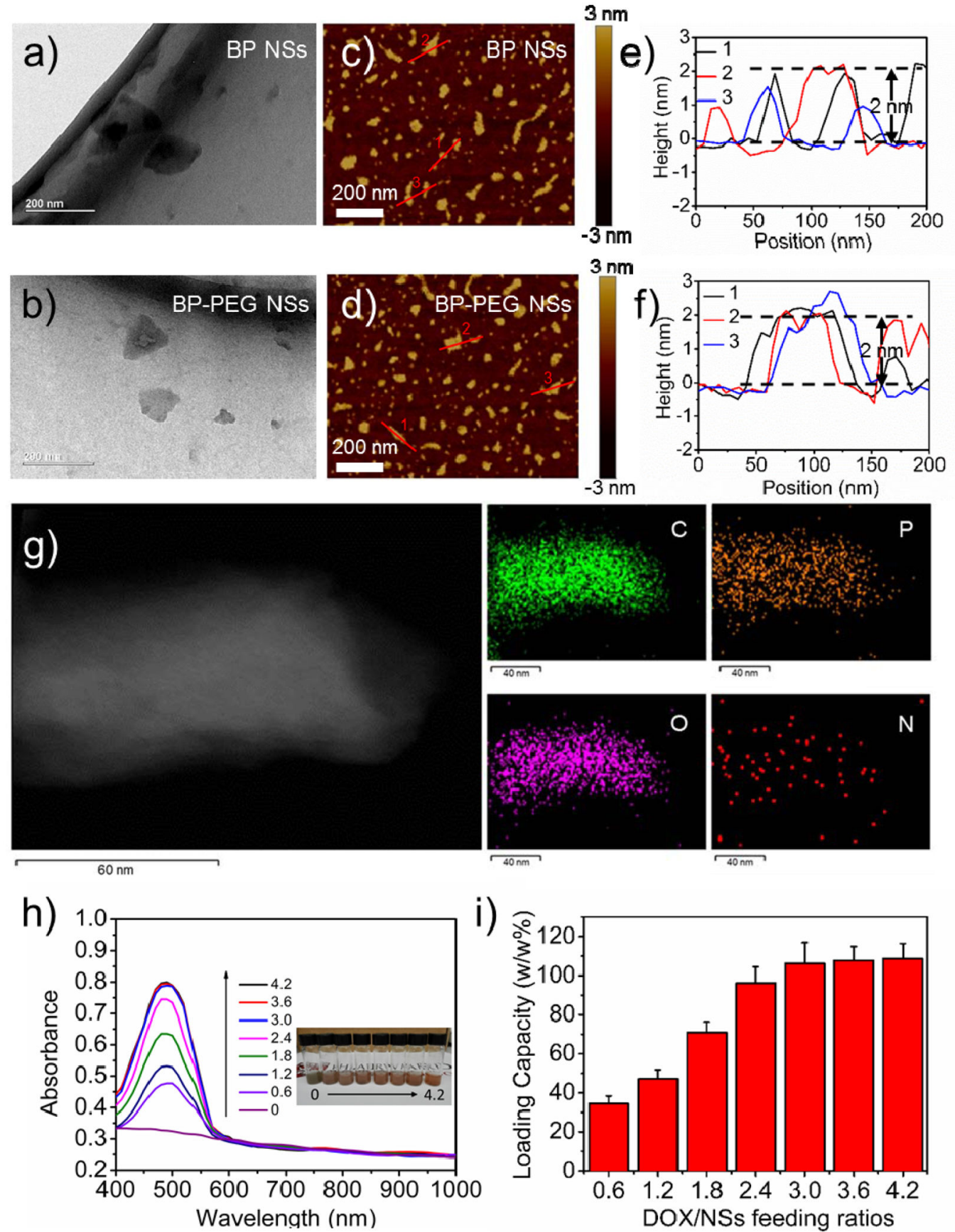

Figure 2.

Characterization of PEGylated BP NSs. TEM images of (a) BP NSs and (b) BP-PEG NSs (scale bar $=200 \mathrm{~nm}$ ). AFM images of (c) BP NSs and (d) BP-PEG NSs (scale bar $=200$ $\mathrm{nm}$ ). AFM measured thickness of (e) BP NSs and (f) BP-PEG NSs. (g) STEM (scale bar = $60 \mathrm{~nm}$ ) and EDS mapping (scale bar $=40 \mathrm{~nm}$ ) of BP-PEG-FA NSs. (h) UV-vis-NIR spectra of BP-PEG/DOX NSs at different DOX/NS feeding ratios after the removal of excess free DOX. (i) DOX loading capacities on BP-PEG NSs (w/w \%) with increasing DOX/NS feeding ratios. 

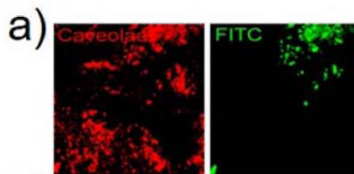

c)

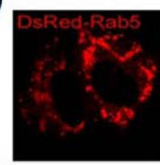

e)
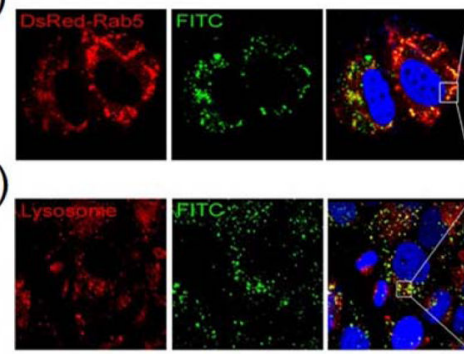

g)

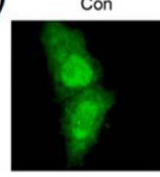

Rapa

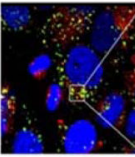

CQ

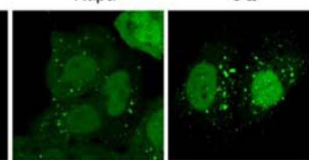

BP
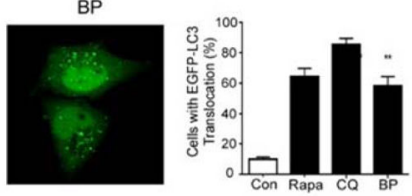

b)
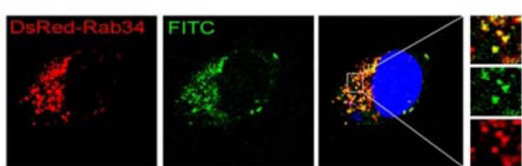

d)

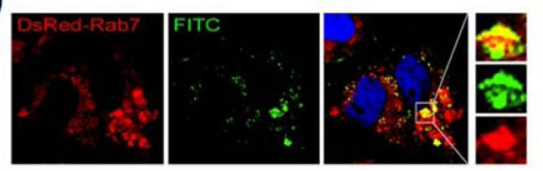

f)

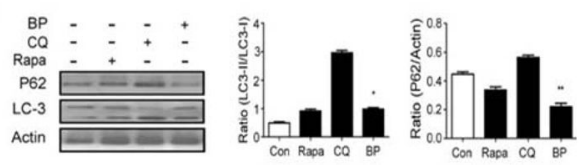

h)

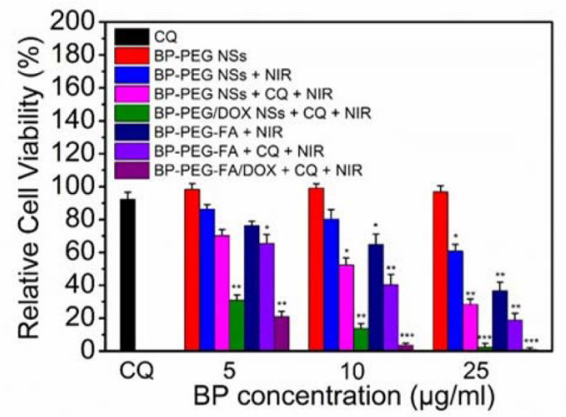

Figure 3.

Endocytosis pathways and biological activities of PEGylated BP NSs. (a) CLSM images of HeLa cells incubated with BP-PEG-FITC NSs for $4 \mathrm{~h}$, while caveolae were detected with primary antibodies against caveolae. CLSM images of HeLa cells transfected with (b) DsRed-Rab34, (c) DsRed-Rab5 and (d) DsRed-Rab7 after $4 \mathrm{~h}$ of incubation with BP-PEGFITC NSs. (e) For lysosome detection, the HeLa cells were treated with BP-PEG-FITC NSs for $4 \mathrm{~h}$ and then were treated with Lyso-Tracker probes for $30 \mathrm{~min}$. (f) HeLa cells were treated with BP-PEG NSs for $24 \mathrm{~h}$, and then the LC3I/II and P62 protein levels were analyzed by western blotting. Histograms represent the quantitative analysis of LC3 and P62 protein expression performed by Image $\mathrm{J}$, respectively ( $* \mathrm{P}<0.05$, ** $\mathrm{P}<0.01)$. (g) EGFPLC3-transfected HeLa cells were treated with BP-PEG NSs for $24 \mathrm{~h}$, and then quantification of cells with EGFP-LC3 vesicles was performed (** $\mathrm{P}<0.01$ ). (h) Relative viabilities of HeLa cells after different types of treatment at different BP concentrations $(5,10,25 \mu \mathrm{g} / \mathrm{ml})$. HeLa cells treated with only BP-PEG NSs or CQ $(30 \mu \mathrm{M})$ was used as control $(* \mathrm{P}<0.05$, $* * \mathrm{P}<0.01, * * * \mathrm{P}<0.001)$. 
a)

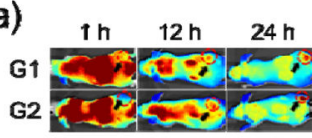

b)

b) $\mathrm{H} \quad$ LI $\mathbf{S}$ LU $\mathrm{K} \quad \mathrm{T}$ $\mathrm{G} 1-\mathrm{CSO} 0$ G2

e)

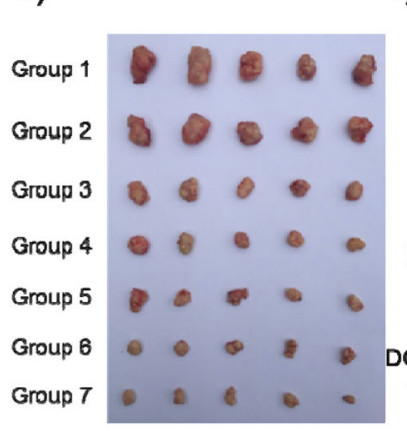

c)

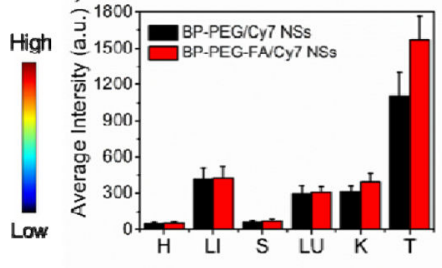

f)

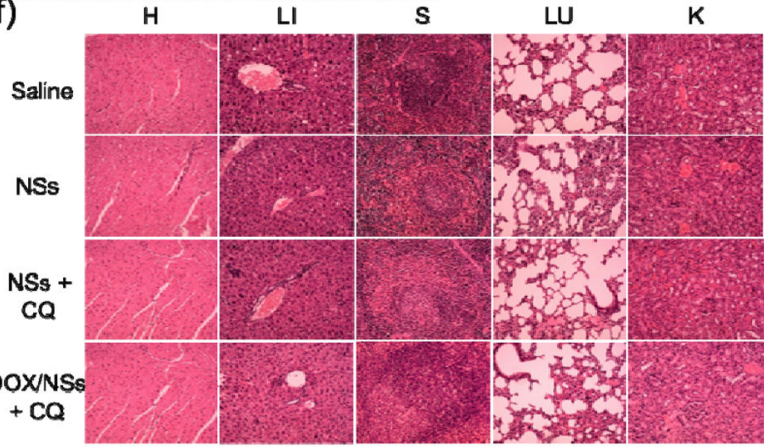

d)

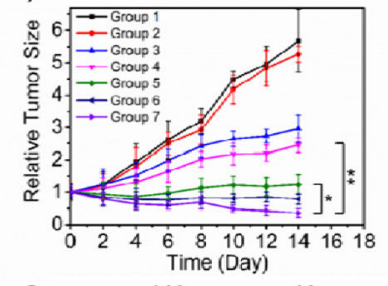

Figure 4.

In vivo NIR imaging and antitumor effect of PEGylated BP NSs. (a) Time-lapse NIR bioimaging of nude mice. The tumors were circled with a red-dotted line. G1: BP-PEG/Cy7 NSs group; G2: BP-PEG-FA/Cy7 NSs group. (b) NIR bio-imaging of major organs and tumors after i.v. injection at 24 h. H: Heart; LI: Liver; S: Spleen; LU; Lung; K: Kidney; T: Tumor. (c) Semi-quantitative biodistribution of BP-PEG/Cy7 NSs and BP-PEG-FA/Cy7 NSs in nude mice measured by the averaged fluorescence intensity of organs and tumors. (d) Inhibition of HeLa tumor growth after different treatments $(* \mathrm{P}<0.05$, ** $\mathrm{P}<0.01)$. Group 1: Saline; Group 2: CQ; Group 3: DOX; Group 4: BP-PEG-FA/DOX; Group 5: BP-PEG-FA + NIR; Group 6: BP-PEG-FA + NIR + CQ; Group 7: BP-PEG-FA/DOX + NIR + CQ. (e) Morphology of tumors removed from the sacrificed mice in all groups at the end point of study. (f) H\&E stained histological images of tissue sections from major organs after 14 days of treatment with BP-PEG-FA NSs, BP-PEG-FA NSs + CQ, and BP-PEG-FA/DOX NSs + CQ. Saline was used as a control. 